\title{
Relationship of Vitamin D Serum Level With Intrauterine Growth Retardation in Pregnant Women
}

\author{
Shohreh Alimohamadi ${ }^{1}{ }^{\mathbb{D}}$, Farzaneh Esna-Ashari $^{1}{ }^{\mathbb{D}}$, Rezvaneh Sadat Beheshti Rooy $^{2^{*} \mathbb{D}}$
}

\begin{abstract}
Objectives: Intrauterine growth retardation (IUGR) is a major problem in midwifery medicine for which no effective treatment has been established. This problem has been associated with significant morbidity and mortality, as well as perinatal mortality. IUGR could be prevented when the treatment is administered before the occurance of irreversible changes. Hence appropriate methods for early detection and successful treatment deem necessary. The purpose of this study was to investigate the relationship of vitamin $\mathrm{D}$ serum level with IUGR in pregnant women.

Materials and Methods: This nested case-control study was conducted on pregnant women who had referred to Fatemieh hospital during 2016. Each of case and control groups included 130 pregnant women. Serum levels of vitamin D were tested in the blood samples obtained from the participants. At the end of the study, all data were analyzed in SPSS software version 16 , and $\chi^{2}$, Fisher exact and $t$ tests were utilized for data analyses. Level of 0.05 was considered statistically significant.

Results: Our results showed that the average age, body mass index at the beginning of the pregnancy, blood group, employment status, and education level were not significantly different between the two groups. Moreover, average serum levels of vitamin $\mathrm{D}$ were about $14.74 \mathrm{ng} / \mathrm{L}$ and $25.34 \mathrm{ng} / \mathrm{L}$ in the case and control groups, respectively. In those with vitamin D deficiency, the chance of IUGR was higher compared to those who had sufficient levels of vitamin D (6.27 times). Therefore, women who had vitamin D deficiency, had almost 6 times more chance of IUGR incidence compared to the women with adequate levels of vitamin $\mathrm{D}(P<0.05)$.

Conclusions: The results verified that serum levels of vitamin D can affect the risk of IUGR; therefore, the incidence of IUGR in babies whose mothers have enough vitamin $\mathrm{D}$ level during pregnancy is lower than that in other babies.

Keywords: Vitamin D, Intrauterine growth retardation, Pregnancy, Hamadan
\end{abstract}

\section{Introduction}

Intrauterine growth restriction (IUGR) is a prevalent disease in pregnancy in which placental insufficiency leads to 5 to 10 times higher mortality and lifelong morbidities (1).

IUGR is a multifaceted problem (2) that increases the risk of hypoxemia, acidemia, preterm deaths, and maternal distress, and disposes the infant to a number of metabolic disorders, polycythemia, lung problems, intraventricular hemorrhage, cognitive dysfunction, and cerebral palsy, which occur in both term and preterm infants (3). Some of the effective factors on the occurrence of IUGR include: the history of chronic maternal diseases (blood pressure, renal diseases, diabetes, Anemia, etc), higher body mass index (BMI), maternal weight gain during pregnancy, maternal age during pregnancy, occupation, different types of fetal infections and chromosomal abnormalities, birth rank, delivery interval, neonatal sex, age of the uterus, placental abruption, and placenta previa (4).

Vitamin D deficiency is highly prevalent among pregnant women (5). One study in Iran has shown that the vitamin $\mathrm{D}$ deficiency level is $69.3 \%$ in pregnant women (6) which is higher than that in other women (7).
Not only vitamin D deficiency during pregnancy causes maternal and fetal side effects (8), it also increases the risk of preeclampsia, gestational diabetes, preterm birth, and birth of a child younger than small for gestational age (SGA) (9), inadequate fetal immune system, wheezing and eczema, and risk of respiratory infections in the infants $(10,11)$.

Some studies have shown the association of vitamin D deficiency with IUGR $(12,13)$. Due to the limitation in the studies on the relationship between vitamin D and IUGR levels, adverse effects of IUGR in pregnancy, and a lack of study in this regard in Iran, this study investigated the relationship between serum levels of vitamin D and IUGR in pregnant women of Hamadan, Iran.

\section{Materials and Methods}

This nested case-control study was conducted on 260 pregnant women who had referred to Fatemieh hospital in Hamadan, Iran during 2018.

In terms of inclusion criteria, singleton pregnant women with a gestational age greater than 36 weeks without the history of underlying diseases, such as diabetes, hypertension, heart disease, and kidney disease, who gave 
birth to a neonate without major congenital anomalies and absent or reverse end-diastolic flow in the case of IUGR embryos, were included in the study. In the case group, there were a total of 130 women who were diagnosed with fetal growth restriction on the basis of clinical findings and then on ultrasound (i.e., the fetal weight below the 10th percentile for a baby's gestational age) based on the intrauterine growth curve of Hadlock et al (14) and also those who were diagnosed with SGA based on the abdominal circumference. They were compared to 130 women with healthy newborns as the control group.

In the control group, pregnant women with embryos weighing less than $2500 \mathrm{~g}$ and more than $4000 \mathrm{~g}$ at birth were excluded from the study. While in the case group, woman with babies weighing more than $2500 \mathrm{~g}$ after birth were excluded.

In this study, based on the estimates made at the beginning of pregnancy, the blood samples were taken from about 1000 pregnant women who had referred to the Fatemieh hospital and were frozen and kept in good conditions, but no specific test was performed on them. At the end of pregnancy, the blood samples of the case and control groups were tested and their vitamin D serum levels were determined.

The data gathering tool in this study was a two-part check list, first part of which consisted of demographic characteristics and BMI. Maternal BMI was determined and documented based on the weight written in the form of prenatal care at the beginning of pregnancy. In the second part of the questionnaire, the obtained amount of vitamin D from the blood test was documented. Blood samples were taken from all study participants to measure the level of vitamin D, and the level of 25 hydroxy vitamin $\mathrm{D}$ was measured in the laboratory by radioimmunoassay.

\section{Data Analysis}

Statistical analysis was performed by SPSS software, version 18.0. Data were analyzed using descriptive statistical chisquare test and Fisher exact test, independent $t$ test, and odds ratio. The significance level was determined less than 0.05 .

\section{Results}

In this nested case-control study, 130 pregnant women with intrauterine growth retardation (IUGR) neonates were compared with other 130 pregnant women with healthy newborns in terms of having IUGR as the control group. The mean age of women in the case and control groups were 26.27 and 25.8 years, respectively, though the difference was not statistically significant. Therefore, the two groups were comparable based on the mean age and BMI at the beginning of pregnancy (Table 1).

Mean vitamin $\mathrm{D}$ levels in the case and control groups were $14.74 \mathrm{ng} / \mathrm{L}$ and $25.34 \mathrm{ng} / \mathrm{L}$, respectively; the difference was statistically significant. Moreover, the mean weight in the pregnant women during pregnancy significantly changed in both groups, while the women in the case group gained less weight ( $9.75 \mathrm{vs.} 10.5 \mathrm{~kg}$ ). The average length of pregnancy in the case group was 254.75 days, while it was higher and equal to 266.13 days in the control group, reaching a significant level (Table 1).

In this study, comparing the level of education between the case and control groups showed no statistically significant difference (Figure 1A).

Considering the occupation of the participants, the majority of subjects in both groups were housewives and in this regard, the two groups were not significantly different. From 130 women in the case group, 16 (12.3\%) were employees, while in the control group, only 2 (1.5\%) were employed. The difference between these two groups, in this respect, was statistically significant (Figure 1B). In general, there was no statistically significant difference between these two groups in terms of occupational status.

In terms of different blood groups, no significant difference was observed between the two groups. Furthermore, no significant relationship was observed between any of different blood groups and the studied subjects (Figure 1C).

Based on the results of the present study, the level of vitamin D classification was significantly different between both case and control groups, such that only 10 (7.7\%) had sufficient vitamin D levels in the case group, while this number was equal to $36(27.7 \%)$ in the control group. In general, there was a significant difference between the two groups in terms of vitamin D serum levels, and the number of cases with vitamin $\mathrm{D}$ deficiencies among women with IUGR neonates or embryos was significantly higher than that in the control group (Figure 1D).

Odds ratios of insufficiency and deficiency in vitamin $\mathrm{D}$ levels in the case and control groups were 1.40 and 6.81,

Table 1. Comparison of the Mean of Baseline Variables in 2 Study Groups

\begin{tabular}{|c|c|c|c|}
\hline Variable & $\begin{array}{c}\text { Control group }(n=130) \\
\text { Mean } \pm \text { SD }\end{array}$ & $\begin{array}{c}\text { Case group }(n=130) \\
\text { Mean } \pm S D\end{array}$ & $P$ Value \\
\hline Age (y) & $25.8 \pm 4.75$ & $26.72 \pm 4.97$ & 0.079 \\
\hline $\mathrm{BMI}\left(\mathrm{kg} / \mathrm{m}^{2}\right)$ & $22.74 \pm 2.64$ & $24.12 \pm 2.87$ & 0.60 \\
\hline Vitamin D (ng/L) & $25.34 \pm 16.09$ & $14.74 \pm 11.85$ & $0.001 *$ \\
\hline The gained weight $(\mathrm{kg})$ & $10.50 \pm 2.33$ & $9.75 \pm 2.42$ & $0.001 *$ \\
\hline Duration of Pregnancy (wk) & $266.13 \pm 17.25$ & $254.75 \pm 10.15$ & $0.001 *$ \\
\hline
\end{tabular}

Note. SD: standard deviation.

* Significant at the 0.05 level $(P<0.05)$. 

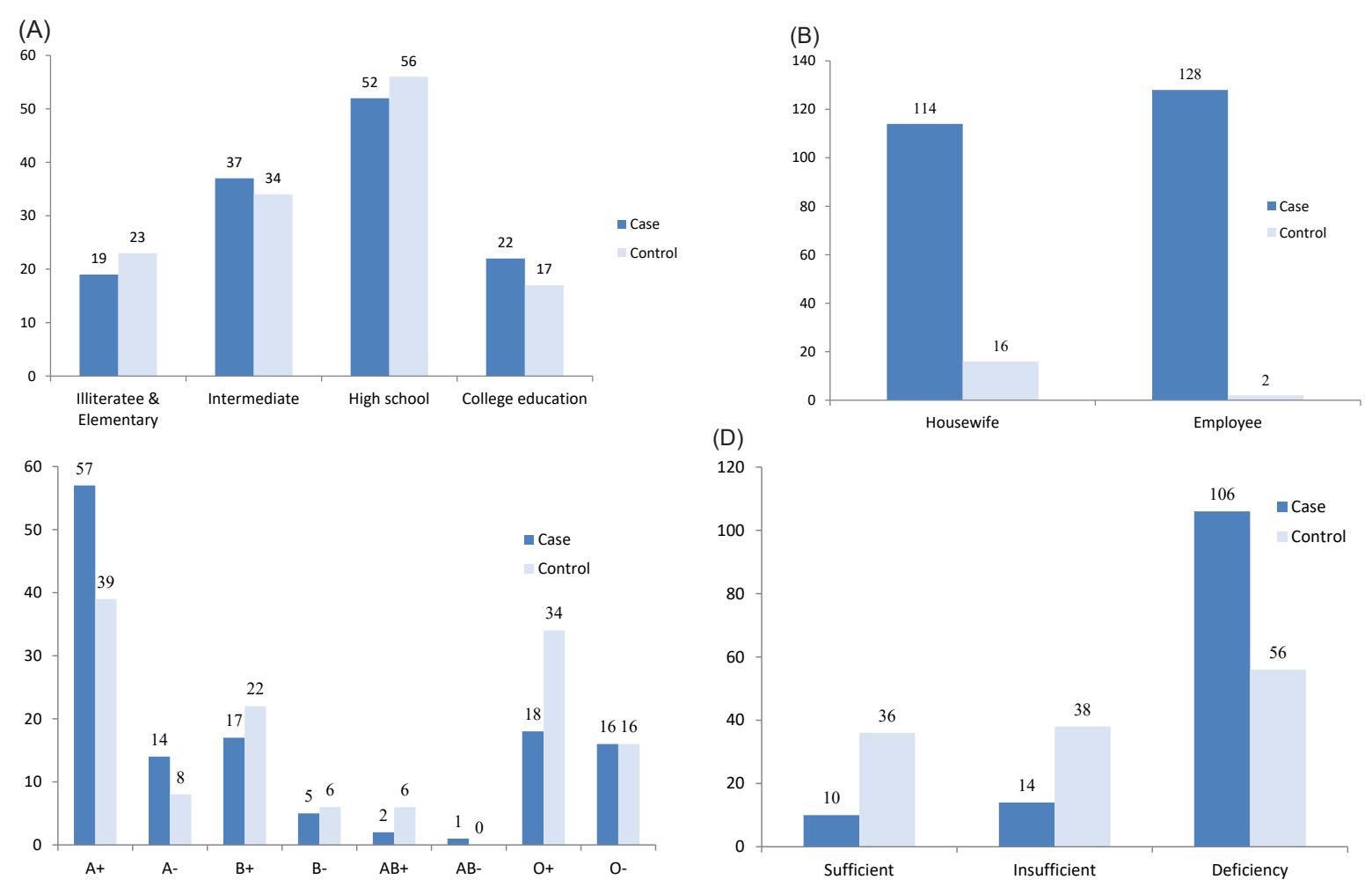

Figure 1. Frequency of (A) Education, (B) Occupation, (C) Blood Groups, and (D) Classified Vitamin D in the Case and Control Groups.

respectively (Table 2 ).

The results of the present study indicated that the infant's sex had no effect on vitamin D level, as vitamin D levels in both sexes were lower in the case group compared with that in the control group (Figure 2A).

Based on the results of this study, sufficient vitamin $\mathrm{D}$ levels were lower in the case group compared with the control group in terms of occupational status in both housewives and employees, however no significant relationship was found between the employment and vitamin D deficiency in the employed women (Figure 2B).

Moreover, according to the results of the present study, BMI did not affect vitamin D levels, and in both normal and obese BMI states, the sufficient vitamin D level was lower in the case group compared with the control group (Figure 2C).

According to the results, the education level was effective on vitamin $\mathrm{D}$ levels and in both educational levels, the level of sufficient vitamin $\mathrm{D}$ was lower in the case group compared with the control group, however no significant relationship was found between the level of education and vitamin D deficiency in the employed low-educated and illiterate participants (Figure 2D).

Additionally, the results of the present study represented that the maternal blood group ( $\mathrm{Rh}$ ) had no effect on vitamin D levels, as in both blood groups, the level of sufficient vitamin D in the case group was lower than that in the control group (Figure 2E).

\section{Discussion}

IUGR is a major problem in midwifery medicine, which is associated with morbidity and perinatal mortality (15).

In our study, the mean gained weight during pregnancy and the mean duration of pregnancy was significantly lower in the case group, compared with the control group.

An important factor in IUGR is maternal weight gain during pregnancy. The direct relationship between the weight gain during pregnancy and the birth weight of the newborn has been shown in many studies $(16,17)$.

Hasan et al found that inadequate maternal weight gain in the third trimester increases the risk of IUGR (18). The study of Margerison-Zilko et al also showed that one kilogram increase in third trimester was associated with a $10 \%$ reduction in the risk of SGA $<10$ th percentile (19).

Table 2. The Calculated Odds Ratio Based on the Level of Classified Vitamin D

\begin{tabular}{lccc}
\hline Vitamin D & Case Group & Control Group & OR (95\% CI) \\
\hline Sufficient $>32$ & 10 & 36 & 1.00 \\
Insufficient (20-32) & 14 & 38 & $1.40(3.0,56.55)$ \\
Deficiency $(<20)$ & 106 & 56 & 0.48 \\
\hline
\end{tabular}

* Significant at the 0.05 level $(P<0.05)$. 

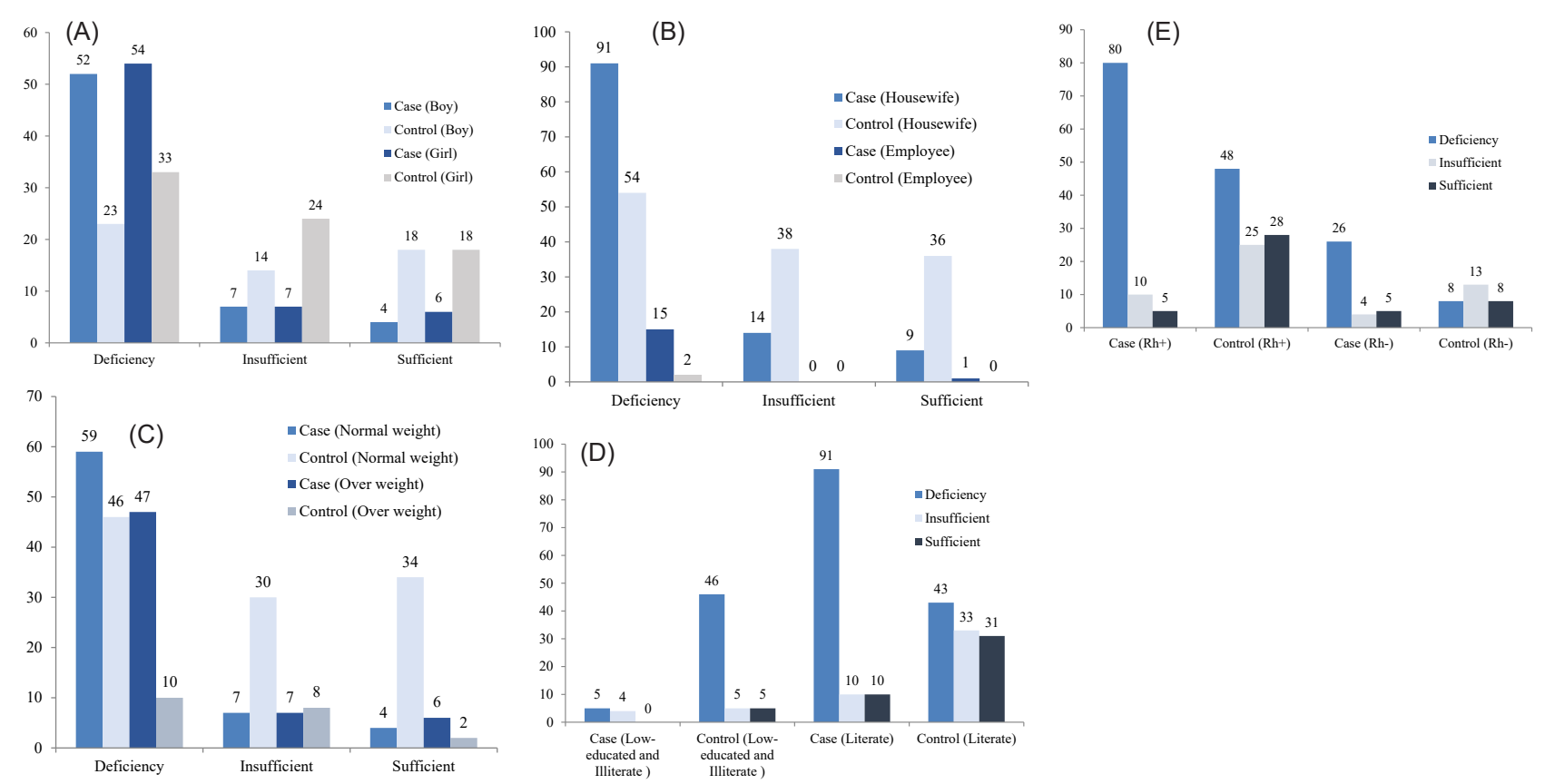

Figure 2. Vitamin D Deficiency in Terms of (A) Gender, (B) Occupational Status, (C) Maternal Weight, (D) Education, (E) Rhesus factor (RH) in Both Groups.

In the study by Reichrath and Querings, vitamin D level during the pregnancy in women with low birth weight infants was significantly lower than its level in other women. They eventually found that vitamin D level in pregnancy was effective in bone metabolism and growth process of the fetus (20). Moreover, in the study by Robinson et al, vitamin D levels in the mothers with low birth weight infants were significantly lower than those in mothers with no IUGR (21). In another study by Gernand et al (13), maternal vitamin D level in the second trimester of the pregnancy was related to the risk of SGA in the white and non-obese women, while this relationship was not seen in the black or obese women. SGA and IUGR are likely associated with weight at birth below $2500 \mathrm{~g}$ (22).

A study reported that vitamin D deficiency at birth was not associated with placental inflammation or neonatal infection among infants with very low birth weight (23).

In the present study, the mean level of vitamin $\mathrm{D}$ in the case group was significantly lower than that in the control group . Vitamin D level was also significantly different between both case and control groups.

In our study, IUGR incidence was significantly different between the case and control groups, to such a level that women with vitamin D deficiency had an IUGR incidence of approximately six times greater than that of women with a sufficient level of vitamin D.

Vitamin D deficiency during pregnancy causes potentially harmful implications in the mother and the fetus $(10,11)$. Several studies have referred to the relationship between vitamin $\mathrm{D}$ deficiency and the incidence of IUGR $(12,13,24)$.

Some studies have shown that increasing the amount of 25-hydroxy vitamin $\mathrm{D}$ in the bloodstream before and during pregnancy contributes to nesting and causes stability in pregnancy, as well as increased calcium uptake required for fetal growth and development (25). Studies have also indicated that the decreased expression of vitamin $\mathrm{D}$ receptors results in functional impairment and limitation in the beneficial effects of vitamin $D$ in regulating the fetus-placental growth $(20,26)$. In the study of Hutabarat et al, maternal vitamin $\mathrm{D}$ deficiency was observed in all pathological pregnancies with a decrease in the staining levels of placental VDR in IUGR (27).

As Zhang et al have shown, severe vitamin D deficiency may play an important role in placental inflammation, which in turn may lead to a higher risk of IUGR and other neonatal side effects (28).

The influence of taking vitamin D supplements during pregnancy in women with vitamin $\mathrm{D}$ deficiency for reducing the adverse effects of IUGR is recommended in future clinical trials. In this study, the correlation between vitamin D deficiency and IUGR was approved; hence, the use of vitamin D supplements before and during pregnancy could be the clinical application of this research.

This study suffers from some limitations. Considering the large sample reservoir (1000 cases) in the beginning of the study, the cost of preparing the kits and keeping the samples at the appropriate temperature were limitations of this study.

\section{Conclusions}

The results of the current study indicated that vitamin D serum levels could be effective on the incidence of IUGR. Moreover, it was shown that the incidence of IUGR in the 
infants whose mothers had sufficient levels of vitamin D during pregnancy was lower than that in other infants. Therefore, it could be predicted the occurrence of IUGR by measuring the vitamin $\mathrm{D}$ serum levels in the early pregnancy and prevented its occurrence by using possible methods.

\section{Conflict of Interests}

The authors declare that they have no conflict of interests.

\section{Ethical Issues}

The study was approved by the Ethics Committee of Hamadan University of Medical Sciences (ID: IR.UMSHA. REC.1394.422). Moreover, an informed consent was obtained from the participants before the study.

\section{Financial Support}

This article was derived from a thesis in Medicine, in Faculty of Medicine, Hamadan University of Medical Sciences (thesis number: 9505122860). The study was financially supported by the Vice-chancellor for Research and Technology of Hamadan University of Medical Sciences.

\section{Acknowledgments}

The authors gratefully acknowledge all personnel in Fatemieh hospital in Hamadan province who helped in data collection.

\section{References}

1. Saw SN, Low JYR, Mattar CNZ, Biswas A, Chen L, Yap CH. Motorizing and optimizing ultrasound strain elastography for detection of intrauterine growth restriction pregnancies. Ultrasound Med Biol. 2018;44(3):532-543. doi:10.1016/j. ultrasmedbio.2017.12.007

2. ACOG. Intrauterine growth restriction. ACOG practice bulletin. 2000;12:324-325.

3. Jones JN, Gercel-Taylor C, Taylor DD. Altered cord serum lipid levels associated with small for gestational age infants. Obstet Gynecol. 1999;93(4):527-531. doi:10.1016/s00297844(98)00489-x

4. Cunningham F, Macdonald P, Gant N. Prenatal care. In: Williams Obstetrics. USA: Appleton Lange 2005.

5. Hong-Bi S, Yin $\mathrm{X}$, Xiaowu $\mathrm{Y}$, et al. High prevalence of vitamin $\mathrm{D}$ deficiency in pregnant women and its relationship with adverse pregnancy outcomes in Guizhou, China. J Int Med Res. 2018;46(11):4500-4505. doi:10.1177/0300060518781477

6. Mirbolouk F, Pakseresht S, Asgharnia M, Farjadmand BM, Kazemnezhad E. Study of vitamin D status in pregnant women in North of Iran. Int J Women's Health Reprod Sci. 2016;4(4):176-180. doi:10.15296/ijwhr.2016.39

7. Shohani M, Mansouri A, Azami M, Soleymani A, Badfar G. The study of vitamin D status in population referred to clinical laboratories in Ilam, West of Iran. Crescent J Med Biol Sci. 2018;5(2):115-118.

8. Larqué E, Morales E, Leis R, Blanco-Carnero JE. Maternal and foetal health implications of vitamin D status during pregnancy. Ann Nutr Metab. 2018;72(3):179-192. doi: $10.1159 / 000487370$

9. Wei SQ, Qi HP, Luo ZC, Fraser WD. Maternal vitamin D status and adverse pregnancy outcomes: a systematic review and meta-analysis. J Matern Fetal Neonatal Med. 2013;26(9):889-899. doi:10.3109/14767058.2013.765849

10. Dawodu A, Akinbi H. Vitamin D nutrition in pregnancy: current opinion. Int J Womens Health. 2013;5:333-343. doi:10.2147/ijwh.s34032

11. Schöttker B, Ball D, Gellert C, Brenner H. Serum 25-hydroxyvitamin D levels and overall mortality. A systematic review and meta-analysis of prospective cohort studies. Ageing Res Rev. 2013;12(2):708-718. doi:10.1016/j. arr.2012.02.004

12. Bodnar LM, Catov JM, Zmuda JM, et al. Maternal serum 25-hydroxyvitamin D concentrations are associated with small-for-gestational age births in white women. J Nutr. 2010;140(5):999-1006. doi:10.3945/jn.109.119636

13. Gernand AD, Simhan HN, Caritis S, Bodnar LM. Maternal vitamin D status and small-for-gestational-age offspring in women at high risk for preeclampsia. Obstet Gynecol. 2014;123(1):40-48. doi:10.1097/aog.0000000000000049

14. Reboul Q, Delabaere A, Luo ZC, et al. Prediction of small-for-gestational-age neonate by third-trimester fetal biometry and impact of ultrasound-delivery interval. Ultrasound Obstet Gynecol. 2017;49(3):372-378. doi:10.1002/uog.15959

15. Scott JR, Gibbs RS, Karlan BY. Danforth's Obstetrics and Gynecology. 10th ed. London: Lippincott Williams \& Wilkins; 2003.

16. Creasy R, Resnik R. Maternal nutrition. In: Maternal Fetal Medicine. 3rd ed. Philadelphia: Saunders; 1994.

17. Abrams B, Selvin S. Maternal weight gain pattern and birth weight. Obstet Gynecol. 1995;86(2):163-169. doi:10.1016/0029-7844(95)00118-b

18. Hasan SMT, Khan MA, Ahmed T. Inadequate maternal weight gain in the third trimester increases the risk of intrauterine growth restriction in rural Bangladesh. PLoS One. 2019;14(2):e0212116. doi:10.1371/journal. pone. 0212116

19. Margerison-Zilko CE, Shrimali BP, Eskenazi B, Lahiff M, Lindquist AR, Abrams BF. Trimester of maternal gestational weight gain and offspring body weight at birth and age five. Matern Child Health J. 2012;16(6):1215-1223. doi:10.1007/ s10995-011-0846-1

20. Reichrath J, Querings K. Vitamin D deficiency during pregnancy: a risk factor not only for fetal growth and bone metabolism but also for correct development of the fetal immune system? Am J Clin Nutr. 2005;81(5):1177; author reply 1177-1178. doi:10.1093/ajcn/81.5.1177

21. Robinson CJ, Wagner CL, Hollis BW, Baatz JE, Johnson DD. Maternal vitamin D and fetal growth in early-onset severe preeclampsia. Am J Obstet Gynecol. 2011;204(6):556.e551554. doi:10.1016/j.ajog.2011.03.022

22. Fang K, He Y, Mu M, Liu K. Maternal vitamin D deficiency during pregnancy and low birth weight: a systematic review and meta-analysis. J Matern Fetal Neonatal Med. 2019:1-7. doi:10.1080/14767058.2019.1623780

23. Puthuraya S, Karnati S, Kazzi SNJ, Qureshi F, Jacques SM, 
Thomas R. Does vitamin D deficiency affect placental inflammation or infections among very low birth weight infants? J Matern Fetal Neonatal Med. 2018;31(14):19061912. doi:10.1080/14767058.2017.1332034

24. Chen $\mathrm{YH}, \mathrm{Fu} \mathrm{L}$, Hao JH, et al. Maternal vitamin D deficiency during pregnancy elevates the risks of small for gestational age and low birth weight infants in Chinese population. J Clin Endocrinol Metab. 2015;100(5):19121919. doi:10.1210/jc.2014-4407

25. Kovacs CS. Vitamin D in pregnancy and lactation: maternal, fetal, and neonatal outcomes from human and animal studies. Am J Clin Nutr. 2008;88(2):520s-528s. doi:10.1093/ ajcn/88.2.520S
26. Robinson CJ, Wagner CL, Hollis BW, Baatz JE, Johnson DD. Maternal vitamin D and fetal growth in early-onset severe preeclampsia. Am J Obstet Gynecol. 2011;204(6):556.e551554. doi:10.1016/j.ajog.2011.03.022

27. Hutabarat M, Wibowo N, Obermayer-Pietsch B, Huppertz B. Impact of vitamin D and vitamin D receptor on the trophoblast survival capacity in preeclampsia. PLoS One. 2018;13(11):e0206725. doi:10.1371/journal.pone.0206725

28. Zhang Q, Chen H, Wang $\mathrm{Y}$, et al. Severe vitamin D deficiency in the first trimester is associated with placental inflammation in high-risk singleton pregnancy. Clin Nutr. 2019;38(4):1921-1926. doi:10.1016/j.clnu.2018.06.978

(c) 2020 The Author(s); This is an open-access article distributed under the terms of the Creative Commons Attribution License (http:// creativecommons.org/licenses/by/4.0), which permits unrestricted use, distribution, and reproduction in any medium, provided the original work is properly cited. 